\title{
3D RECONSTRUCTION OF CITRUS TREES USING AN OMNIDIRECTIONAL OPTICAL SYSTEM
}

\author{
L. F. Castanheiro ${ }^{1,}$ *, A. M. G. Tommaselli ${ }^{1}$, M. B. Campos $^{2}$, A. Berveglieri ${ }^{1}$, G. Santos ${ }^{1}$ \\ ${ }^{1}$ Department of Cartography, São Paulo State University (UNESP) at Presidente Prudente, São Paulo 19060-900, Brazil - \\ guilherhsantos@gmail.com; (leticia.ferrari, a.tommaselli, a.berveglieri)@unesp.br \\ ${ }^{2}$ Department of Remote Sensing and Photogrammetry, Finnish Geospatial Research Institute FGI, Geodeetinrinne 2, FI-02430 \\ Masala, Finland - mariana.campos@ maanmittauslaitos.fi
}

KEY WORDS: Digital agriculture, orange orchard, poly-dioptric system, fisheye lens, dual-camera, terrestrial mapping, camera calibration, 3D tree modelling.

\begin{abstract}
:
This paper presents a feasibility study on the use of omnidirectional systems for 3D modelling of agricultural crops, aiming a systematic monitoring. Omnidirectional systems with multiple sensors have been widely used in close-range photogrammetry (CRP), which can be a good alternative to provide data for digital agriculture management. The GoPro Fusion dual-camera is the omnidirectional system used in this work. This system is composed of two cameras with fisheye lenses that cover more than $180^{\circ}$ each one in back-to-back position. System calibration, camera orientation and 3D reconstruction of an agricultural cultivated area were performed in Agisoft Metashape software. A $360^{\circ}$ calibration field based on coded targets (CTs) from Agisoft Metashape software was used to calibrate the omnidirectional system. The 3D reconstruction of an orange orchard was performed using fisheye images taken with GoPro Fusion. The results show the potential of using an omnidirectional system for 3D modelling in agricultural crops, in particular citrus trees. Interior orientation parameters (IOPs) was estimated using Agisoft Metashape target/software with a precision of $9 \mathrm{~mm}$. A 3D reconstruction model of the orange orchard area was obtained with an accuracy of $3.8 \mathrm{~cm}$, which can be considered acceptable for agricultural purposes.
\end{abstract}

\section{INTRODUCTION}

Digital agriculture can be defined as a farming management technique that combines technologies to analyze a huge amount of data from precision agriculture and other sources, aiming to minimize costs and to increase production. Nowadays, increasing the production with environmental sustainability is one of the main world challenges, which requires new management technologies. Remote sensing data can be used for several agricultural applications, such as soil monitoring (Prosdocimi et al., 2017), mapping diseased and healthy plants (Moriya et al., 2019), and estimation of plant phenotyping (Ravi et al., 2018). Most of the existing works has focused on aerial platforms, mainly with Unmanned Aerial Vehicles (UAV), aiming a systematic and fast acquisition. However, acquisition from the ground perspective can provide complementary information such as plants and soil morphology (shape and structure), especially in fruticulture/horticulture areas, which cannot be assessed by an aerial sensor.

Esau et al. (2018) presented a multi camera system composed of nine digital frame RGB cameras mounted on a tractor. Images were taken and processed in real time to determine where to spray pesticides, aiming a control and uniform application. Sodhi et al. (2017) presented a mobile system composed of ten cameras that move vertically along the plant for $3 \mathrm{D}$ plant phenotyping. Bao et al. (2018) presented a system with 12 RGB cameras to circumvent the wind problem for 3D plant phenotyping.

Images with large field of view (FoV) can be acquired by nonconventional systems, which need a smaller number of images to cover a larger area. An alternative for a systematic data collection from ground perspective in agricultural cultivated areas is the use of fisheye lens cameras, which are seldom explored in the literature. Only few works can be mentioned. Ericson and Astrand (2010) used a fisheye lens and a catadioptric system to assess the proposed method for detecting parallel rows on agricultural crops. Ohi et al. (2018) presented an autonomous pollination robot named BrambleBee, composed of a 3D LiDAR system, a fisheye lens camera and a navigation system were embedded in the robot platform to map bramble plants.

These related works highlighted that a reliable analysis of agricultural areas depends on the quality of the acquired data and the processing chain, which are affected by different sources of errors. Therefore, a feasibility study using fisheye images obtained with the GoPro Fusion omnidirectional system in an orange orchard was performed, aiming the $3 \mathrm{D}$ trees modelling. The omnidirectional system is composed of two fisheye lenses in the same structure (poly-dioptric system - Maas, 2008). Each lens covers more than $180^{\circ}$ in back-to-back position, enabling a $360^{\circ}$ FoV. Agisoft Metashape software was used to the photogrammetric process. The main steps of the photogrammetric process were: (1) camera calibration using a $360^{\circ}$ calibration field based on coded targets (CTs) from Agisoft Metashape software; (2) fisheye image acquisition in an orange crop area and, (3) camera orientation and the 3D reconstruction of orange trees. These steps will be detailed and assessed in the following sections. The results have shown the feasibility of using an omnidirectional system for 3D modelling of an agricultural crop.

\section{BACKGROUNG}

Omnidirectional systems based on multi-camera have gained attention for many close range photogrammetry (CRP)

\footnotetext{
* Corresponding author
} 
applications, e.g. terrestrial mobile mapping, due to the large FoV $\left(360^{\circ}\right)$. An omnidirectional system with multi-camera can be designed with conventional (e.g perspective lens) and nonconventional geometry optical system, such as fisheye lenses. Fisheye lenses have a large FoV, which can be hemispheric $\left(110^{\circ}\right.$ $\left.-180^{\circ}\right)$ or hyper-hemispheric $\left(>180^{\circ}\right)$. Therefore, a full-spherical panorama can be covered using only two fisheye lenses, which enable a compact and lighter design of the omnidirectional systems $\left(360^{\circ} \mathrm{FoV}\right)$.

Fisheye lenses do not follow the perspective projection. Therefore, a suitable mathematical model is required for an automated image-based 3D modelling. The fisheye lenses are usually designed with the projection of the observations from a sphere surface onto an image plane following the equidistant, orthogonal, stereographic or equisolid-angle projections (Schneider et al., 2009). Due to the large radial distortion in fisheye lenses, these mathematical models are combined with distortion corrections such as Conrady-Brown's distortion model (Conrady, 1919; Brown, 1971) or polynomials (Kannala and Brandt, 2006).

According to Abraham and Forstner (2005), most of fisheye lenses follow the equidistant projection. For instance, the equidistant model is used in Agisoft Metashape, and the distortions are modelled with Conrady-Brown's distortion model combined with the affinity and shear parameters (Fraser, 1997). Equation 1 presents the equidistant equations and Equation 2 the distortion models, which are compatible with the mathematical models implemented at Agisoft Metashape software (Agisoft, 2018).

$$
\begin{gathered}
x=-f \frac{X_{C}}{\sqrt{\left(X_{C}\right)^{2}+\left(Y_{C}\right)^{2}}} a \tan \left(\frac{\sqrt{\left(X_{C}\right)^{2}+\left(Y_{C}\right)^{2}}}{Z_{C}}\right)+x_{0}+\Delta x \\
y=-f \frac{Y_{C}}{\sqrt{\left(X_{C}\right)^{2}+\left(Y_{C}\right)^{2}}} a \tan \left(\frac{\sqrt{\left(X_{C}\right)^{2}+\left(Y_{C}\right)^{2}}}{Z_{C}}\right)+y_{0}+\Delta y \\
\Delta x=x\left(K_{1} r^{2}+K_{2} r^{4}+K_{3} r^{6}\right)+\left(P_{1}\left(r^{2}+2 x^{2}\right)+2 P_{2} x y\right)\left(1+P_{3} r^{2}+P_{4} r^{4}\right)+B_{1} x+B_{2} y \\
\Delta y=y\left(K_{1} r^{2}+K_{2} r^{4}+K_{3} r^{6}\right)+\left(P_{2}\left(r^{2}+2 y^{2}\right)+2 P_{1} x y\right)\left(1+P_{3} r^{2}+P_{4} r^{4}\right)
\end{gathered}
$$

where $\mathrm{x}$ and $\mathrm{y}$ are the image coordinates, $\mathrm{f}$ is the focal length, $\mathrm{x}_{0}$ and $\mathrm{y}_{0}$ are principal point offsets, $\mathrm{X}_{\mathrm{C}}, \mathrm{Y}_{\mathrm{C}}$ and $\mathrm{Z}_{\mathrm{C}}$ are point coordinates in the photogrammetric system, and $\Delta \mathrm{x}$ and $\Delta \mathrm{y}$ are the distortion parameters referring to radial symmetric $\left(\mathrm{K}_{1}, \mathrm{~K}_{2}\right.$, $\left.\mathrm{K}_{3}\right)$, decentering $\left(\mathrm{P}_{1}, \mathrm{P}_{2}, \mathrm{P}_{3}, \mathrm{P}_{4}\right)$, affinity and shear distortions parameters $\left(\mathrm{b}_{1}, \mathrm{~b}_{2}\right)$.

\section{PHOTOGRAMMETRIC PROCESS: DATA SET AND EXPERIMENTS}

Scientific and commercial approaches have been developed to adapt the photogrammetric processes, such as camera calibration, rectification and image matching, considering fisheye lens mathematical models (Scaramuzza et al., 2006; Kannala and Brandt, 2006; Marcato Junior et al., 2015; Campos et al., 2019). This section describes the photogrammetric processes used in the Agisoft Metashape software. Section 3.1 summarizes the camera calibration process of the omnidirectional system (dual-fisheye lenses) used (GoPro Fusion). Section 3.2 describes the cameras orientation step and the 3D reconstruction of an orange orchard.

\subsection{Camera Calibration}

Camera calibration is a fundamental step to obtain a reliable 3D reconstruction, aiming high accuracy applications such as precision agriculture. Fisheye camera calibration has been widely discussed in the literature (Abraham and Forstner, 2005; Schneider et al., 2009; Tommaselli et al., 2014; Marcato Junior et al., 2015). However, the dual-fisheye camera calibration started to be discussed recently, especially motivated by the new commercial systems available in the market (e.g. Ricoh Theta, Insta360, Samsung Gear360, Nikon Keymission 360, GoPro360). Multi-camera calibration usually requires the estimation of two sets of IOPs (interior orientation parameters) and EOPs (exterior orientation parameters), as well a set of ROPs (relative orientation parameters). Different approaches to calibrate a dual-fisheye lens camera (Ricoh Theta S) were proposed by Aghayari et al. (2017) and Campos et al. (2018).

In this work, a simultaneous IOPs determination of the GoPro Fusion dual-camera was performed in Agisoft Metashape. Table 1 presents the technical details of the GoPro Fusion system. Agisoft Metashape software uses the EXIF file to extract the camera configuration, but it was provided an inaccurate pixel size value. Then, the pixel size was recalculated considering the sensor and image size, resulting in $0.002 \mathrm{~mm}$.

\begin{tabular}{|l|l|}
\hline \multicolumn{2}{|c|}{ GoPro Fusion Settings } \\
\hline Sensor size & $1 / 2.3 " 6.17 \times 4.55 \mathrm{~mm}(\mathrm{CMOS})$ \\
Dual-fisheye image & $3104 \times 3000$ pixels each image \\
Nominal focal length & $3 \mathrm{~mm}$ \\
Camera dimensions & $4.0 \times 7.5 \times 7.4 \mathrm{~cm}$ \\
Weight & $220 \mathrm{~g}$ \\
Wireless & Wifi, Bluetooth and GPS \\
Pixel size (calculated) & $0.002 \mathrm{~mm}$ \\
\hline
\end{tabular}

Table 1. Technical information of GoPro Fusion.

A total of 26 fisheye images (13 from each sensor) were used for the camera calibration procedure. Convergent and rotated images were taken to avoid correlations between IOPs and EOPs (Fraser, 1997). The $360^{\circ}$ calibration field (Figure 1a) has 160 ArUco

(2) targets (Muñoz-Salinas, 2012) and 100 coded targets (CTs) designed for an automatic detection by Agisoft Metashape with dimensions of $25 \times 25 \mathrm{~cm}$. Only the CTs were used in the calibration procedure, which are automatically detected with subpixel accuracy by Agisoft Metashape. The ground coordinates of 3 CTs $(62,64$ and 65) were used as constraints in the selfcalibration.

Figure 1a shows the images of sensor 1 (S1) and sensor 2 (S2) from GoPro Fusion camera (Figure 1c) acquired at the $360^{\circ}$ calibration field. Figure 1b presents an example of CTs used. Figure 1c shows a lateral and frontal view of the GoPro Fusion camera. The coordinates of ArUco and CTs were estimated considering a local reference system $(\mathrm{X}, \mathrm{Y}, \mathrm{Z})$, as illustrated in Figure 1a. 

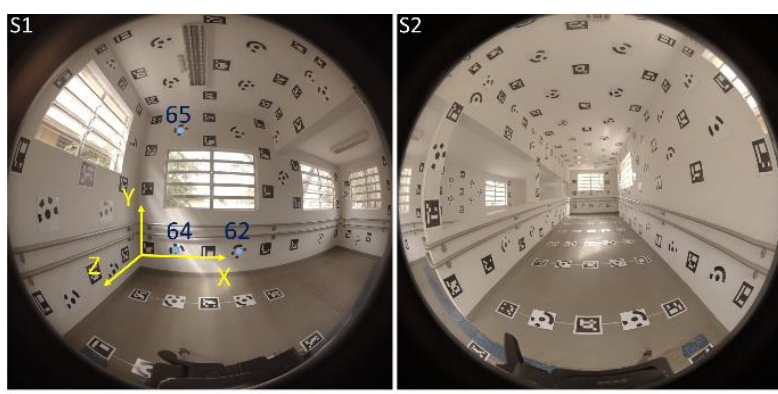

(a)

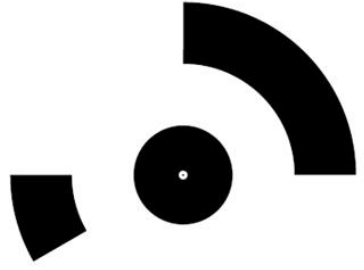

(b)

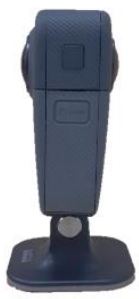

(c)

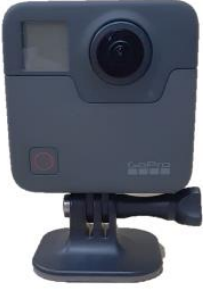

)
Figure 1. (a) Images from sensor 1 (S1) and sensor 2 (S2) of the $360^{\circ}$ camera calibration field, in which the reference system and the 3 CTs used in the self-calibration are illustrated in S1, (b)

Agisoft Metashape/Photoscan coded targets (CTs) and (c) a

lateral and frontal view of the GoPro Fusion camera.

As mentioned, the GoPro Fusion camera has two fisheye lenses that cover more than $180^{\circ}$ (hyper-hemispheric lenses). The overlap between images from $\mathrm{S} 1$ and $\mathrm{S} 2$ can be observed in Figure1a. Campos et al. (2018) show that equidistant projection is suitable only for points in the hemispheric field. Considering that the fisheye mathematical model used in Agisoft Metashape is the equidistant projection, points in the hyper-hemispheric field were not used in the calibration procedure.

The consistency of the camera calibration was analyzed considering the discrepancies between distances directly measured in the $360^{\circ}$ calibration field and distances estimated in the self-calibration. Ten well-distributed horizontal, vertical and diagonal distances were measured in the $360^{\circ}$ calibration field using a $2 \mathrm{~m}$ calliper with precision of $0.02 \mathrm{~mm}$. The distortion parameters considered in camera calibration were the radial symmetric parameters $\left(\mathrm{K}_{1}, \mathrm{~K}_{2}, \mathrm{~K}_{3}\right)$ and two decentring parameters $\left(\mathrm{P}_{1}, \mathrm{P}_{2}\right)$.

The ROPs $\left(\Delta \omega, \Delta \varphi, \Delta \kappa, \mathrm{B}_{\mathrm{X}}, \mathrm{B}_{\mathrm{Y}}, \mathrm{B}_{\mathrm{Z}}\right)$ are not estimated by Agisoft Metashape software. Therefore, ROPs were calculated in an external step considering the estimated sensor poses. The analysis was conducted considering that the distance between the two cameras are stable during the acquisition, excepted by small random errors.

\subsection{D photogrammetric modelling process}

The feasibility study of the omnidirectional system was performed using fisheye images collected in an orange orchard, located at Guacho farm, Agroterenas S/A citrus (Santa Cruz do Rio Pardo - SP, Brazil). The GoPro Fusion camera was positioned over the operator head and images were taken while the operator walks between the orange trees. A total of 116 images (58 dual-fisheye images) were used. 3D coordinates of ten ground points (pre-signalized) were estimated using GPSRTK, which were used as control and check points. Figure 2 shows an example of fisheye images from sensor 1 (S1) and sensor 2 (S2) acquired in the orange orchard.
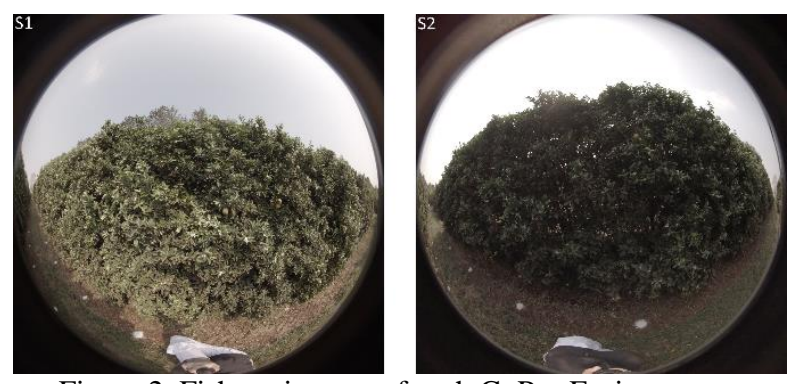

Figure 2. Fisheye images of each GoPro Fusion sensor (sensor1-S1, sensor 2-S2) acquired in the orange orchard.

First, camera configurations were set in the Agisoft Metashape software considering the fisheye model (equidistant model) and the IOPs obtained in the previous camera calibration process described in Section 3.1. The reference system was set as WGS84/UTM, fuse 22S. Six control points were used to estimate the EOPs and four points were considered as check points. A standard deviation of $0.01 \mathrm{~m}$ was considered for both control and check points, since the 3D coordinates were estimated using GPS-RTK. These points were manually identified and measured in the fisheye images with 1 pixel accuracy. Then, images were firstly aligned in high mode generating tie points. These tie points were refined in manual and automatic mode considering an image observation accuracy higher than 2 pixels. Finally, the first estimated sensor poses are improved considering only the refined tie points.

The dense point cloud was generated in ultra-high mode. Different approaches can be used to assess the position accuracy of a point cloud (Lehtola et al., 2017). In this work, four check points, surveyed with GPS-RTK, were used to analyze the 3D position accuracy of the generated point cloud. In addition, the $3 \mathrm{D}$ reconstruction of interest features in agriculture areas, such as trees, fruits and soil, were also verified in the generated dense point cloud. The CloudCompare software was used to visualize the generated dense point cloud and measured tree and fruit features.

\section{RESULTS}

\subsection{Interior Orientation Parameters estimation.}

Table 2 shows the estimated IOPs and the respective standard deviations. The standard deviation of the estimated focal length (f) and the principal point coordinates $\left(\mathrm{x}_{0}, \mathrm{y}_{0}\right)$ were less than 1 pixel $(0.002 \mathrm{~mm})$, as recommended by Campos et al. (2015) for close range photogrammetry applications.

\begin{tabular}{|ccc|}
\hline IOP & Estimated value & Std. deviation \\
\hline $\mathrm{f}(\mathrm{mm})$ & 2.2354 & 0.0011 \\
$\mathrm{X}_{0}(\mathrm{~mm})$ & -0.0110 & 0.0012 \\
$\mathrm{y}_{0}(\mathrm{~mm})$ & -0.0298 & 0.0013 \\
$\mathrm{~K}_{1}$ & $1.32104 \mathrm{e}-001$ & $3.42336 \mathrm{e}-004$ \\
$\mathrm{~K}_{2}$ & $-1.58702 \mathrm{e}-002$ & $7.91194 \mathrm{e}-005$ \\
$\mathrm{~K}_{3}$ & $5.62292 \mathrm{e}-003$ & $8.45114 \mathrm{e}-006$ \\
$\mathrm{P}_{1}$ & $-1.04975 \mathrm{e}-005$ & $2.74641 \mathrm{e}-004$ \\
$\mathrm{P}_{2}$ & $-1.47747 \mathrm{e}-004$ & $3.02222 \mathrm{e}-004$ \\
\hline
\end{tabular}

Table 2. Estimated values of IOPs and the standard error.

The RMSE of discrepancies between control and estimated distances was $9 \mathrm{~mm}$. The GSD (ground sample distance) of the fisheye images acquired in the calibration field varies from 1 to $8 \mathrm{~mm}$, therefore, the calibration results can be considered consistent. Improvements in the system calibration technique can 
be done including, for example, stability constrains. The average value of estimated distances between front and back cameras was $3.3 \mathrm{~cm}$, which is compatible with the estimated sensor physical separations. The average of estimated ROPs are presented in Table 3

\begin{tabular}{|ccccccc|}
\hline$\Delta \omega\left(^{\circ}\right)$ & $\Delta \varphi\left({ }^{\circ}\right)$ & $\Delta \kappa\left(^{\circ}\right)$ & $\begin{array}{c}\mathbf{B x} \\
(\mathbf{c m})\end{array}$ & $\begin{array}{c}\mathbf{B y} \\
(\mathbf{c m})\end{array}$ & $\begin{array}{c}\mathbf{B z} \\
(\mathbf{c m})\end{array}$ & $\mathbf{D}_{\mathbf{P C}}(\mathbf{c m})$ \\
\hline-0.13 & -0.15 & 179.13 & 1.50 & 1.13 & 2.33 & 3.35 \\
\hline
\end{tabular}

Table 3. Average calculated values of ROPs components $(\Delta \omega$,

$\Delta \varphi$ and $\Delta \kappa$ in degree and $\mathrm{BX}, \mathrm{BY}$ and $\mathrm{Bz}$ in centimeter).

\subsection{D crop reconstruction.}

The errors in control and check points comparing the estimated and the ground values were $3.2 \mathrm{~cm}$ and $3.8 \mathrm{~cm}$, respectively. These errors are compatible with GPS-RTK accuracy and suitable for agricultural applications. A reprojection error of 0.7 pixels in the image coordinates was obtained in both control and check points, which is consistency with the image measurement error. The average of the distances between both sensors was $3.8 \mathrm{~cm}$, which is compatible with the ROPs calculated in the calibration. The generated dense point cloud of orange orchard is presented in Figure 3. Figure 3a presents a top view of the generated 3D crop modelling and the green lines represent the cross sections. Figure $3 \mathrm{~b}$ shows the longitudinal and transversal cross sections.
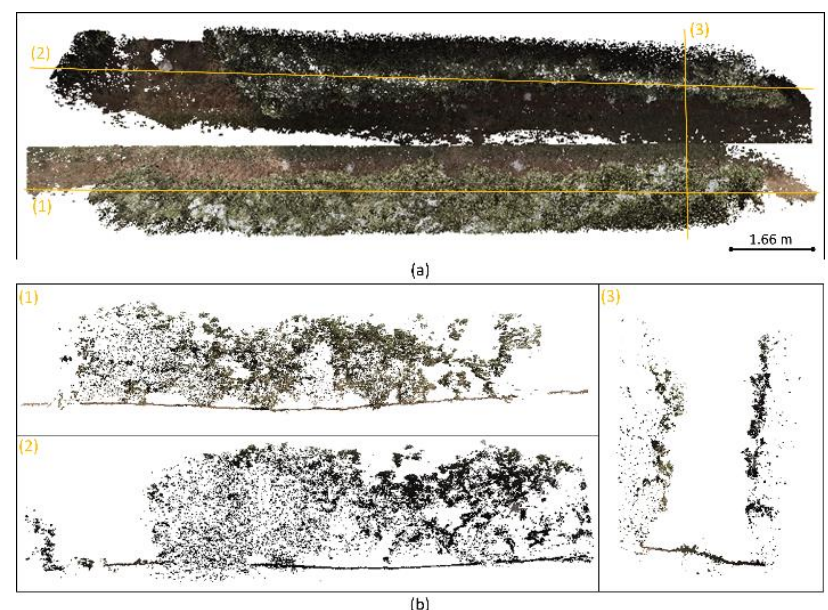

Figure 3. 3D reconstruction of the orange orchard: (a) top view of dense point cloud and cross section in green lines, and (b) longitudinal and transversal cross sections.

Interest features for agriculture can be obtained from dense point clouds, such as tree heights, volume, density, leaf area, ground cover, trees spacing and orange fruit diameter. As examples, Figure 4a shows a tree height measurement in the generated point cloud using CloudCompare. A Canopy Height Model could also be generated by subtracting the treetops from the ground height. Figure $4 \mathrm{~b}$ presents an example of the $3 \mathrm{D}$ reconstruction of a single orange fruit, the diameter measurement of it and the fitted sphere option in CloudCompare software. Table 4 shows the measurements of ten tree heights, which are compared with the heights directly measured in the field. Four diameters of orange fruits were manually measured in the generated point cloud, as presented in Figure $4 \mathrm{~b}$. In addition, spheres were fitted for each orange point cloud in CloudCompare (Figure 4b). Table 5 presents the diameters that were manually measured and automatically estimated by sphere adjustment, as well as the estimated precision $(\sigma)$ of the fitted spheres. The diameters measured in the generated point cloud are compared of the fruit diameters in the orange orchard, which values are around $6 \mathrm{~cm}$.
Other parameters can be extracted from the dense point cloud, such as leaf area, canopy roughness and volume. Furthermore, exposed soil can be detected from classification of dense point cloud.

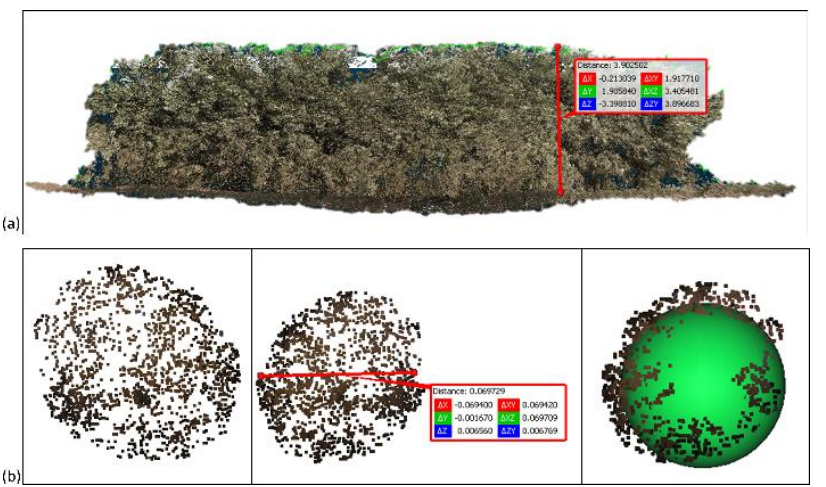

Figure 4. Examples of (a) a tree height measurement in the point cloud and (b) the 3D reconstruction of an orange fruit, the diameter measurement and the fitted sphere in CloudCompare.

\begin{tabular}{|cc|}
\hline Tree & Height $(\mathbf{m})$ \\
\hline 1 & 4.036 \\
2 & 3.636 \\
3 & 3.902 \\
4 & 3.608 \\
5 & 3.863 \\
6 & 3.696 \\
7 & 3.519 \\
8 & 3.830 \\
9 & 3.774 \\
10 & 3.883 \\
\hline
\end{tabular}

Table 4. Tree height measured in the generated dense point cloud.

\begin{tabular}{|c|c|c|}
\hline Fruit & Manual diameter $(\mathbf{c m})$ & $\begin{array}{c}\text { Fitted sphere }(\mathbf{c m}) \\
\text { Diameter/ } \mathbf{\sigma}\end{array}$ \\
\hline 1 & 6.9 & $7.1 / 0.69$ \\
\hline 2 & 6.3 & $6.2 / 0.79$ \\
\hline 3 & 5.8 & $5.9 / 0.78$ \\
\hline 4 & 7.1 & $7.3 / 0.84$ \\
\hline
\end{tabular}

Table 5. Orange diameters manually measured in the generated point cloud and diameters obtained from the fitted spheres.

\section{CONCLUSION}

This paper presented a feasibility study of a low-cost omnidirectional system (GoPro Fusion) for orange orchard mapping. The results showed the potential of an omnidirectional system for 3D modelling of agricultural crops, achieving centimetre accuracy in the check points. Important attributes for digital agriculture can be accurately obtained from a terrestrial dense point cloud, such as tree heights and fruit measurements, which sometimes are not available from an aerial perspective. On the other hand, the tops of high trees can be occluded in terrestrial perspective. Therefore, a full-mapping of the agricultural crops can be achieved combining multi-sensor data, such as aerial images, LiDAR data and multispectral and hyperspectral images.

The GoPro Fusion is a low-cost sensor and the use of two fisheye lenses make it a light-weight omnidirectional system, which can be integrated in a portable mobile mapping system. The recent advances both in camera hardware and sensor models, have enabled the development of mobile mapping alternatives using 
omnidirectional system. A system with fisheye lenses can be a feasible choice, in which a low number of images are necessary compared to perspective cameras. The large FoV can be considered more important than high resolution with perspective image for the feasibility of mobile mapping application, as discussed by Caruso et al. (2015). The benefits on using large FoV images were presented in details by Zhang et al. (2016). Therefore, fisheye models have been implemented in scientific and commercial software due to the growing use of fisheye images for accurate applications, which can be considered a trend in CRP. In this direction, in our work we have used a friendlyuser commercial software. Agisoft Metashape can be considered a suitable software for 3D reconstruction with fisheye images for agricultural purpose showing the potential of the use of large FoV images by non-specialist users.

However, some limitations when using a dual-fisheye camera can be highlighted. Some concerns about fisheye geometry treatment still remain, mainly for dual-fisheye cameras. The equidistant fisheye model, implemented in Agisoft Metashape, is not suitable for objects projected in the hyper-hemispheric image field. To circumvent this limitation, Song et al. (2018) used equidistant projection combined with a polynomial model. The use of hyperhemispheric points requires further investigations, which will be discussed in future works. Campos et al. (2018) presented improvements in the calibration of a dual-fisheye system when considering stability of ROPs as constraints. ROPs cannot be used as constraints in Agisoft Metashape software, which can be suggested as a future work to improve the calibration results.

\section{ACKNOWLEDGEMENTS}

This study was funded by the Coordenação de Aperfeiçoamento de Pessoal de Nível Superior - Brasil (CAPES) - Finance Code 001 (Grants: 88881. 310314/2018-01 and 88882.433941/201901), Conselho Nacional de Desenvolvimento Científico e Tecnológico - CNPq (Grant: 155739/2018-2 and 150306/2018$0)$. The authors are thankful AGT-Bravium-Fundunesp for all support they offered to develop field campaigns.

\section{REFERENCES}

Abraham, S., Förstner, W., 2005. Fish-eye-stereo calibration and epipolar rectification. ISPRS Journal of photogrammetry and remote sensing, v. 59, n. 5, p. 278-288. doi.org/10.1016/j.isprsjprs.2005.03.001

Aghayari, S., Saadatseresht, M., Omidalizarandi, M., Neumann, I., 2017. Geometric calibration of full spherical panoramic Ricoh-Theta camera. In: ISPRS Annals of the Photogrammetry, Remote Sensing and Spatial Information Sciences, v. 4, p. 237. doi.org/10.5194/isprs-annals-iv-1-w1-237-2017

Agisoft, L. L. C. Agisoft Metashape user manual, Professional edition, Version 1.5. Agisoft LLC, St. Petersburg, Russia, from https://www. agisoft. com/pdf/metashape-pro_1_5_en.pdf. (2 July 2019).

Bao, Y., Tang, L., Breitzman, M. W., Salas Fernandez, M. G., Schnable, P. S., 2019. Field-based robotic phenotyping of sorghum plant architecture using stereo vision. Journal of Field Robotics, 36(2), 397-415. doi.org/10.1002/rob.21830

Brown, D. C., 1971. Close-range camera calibration. Photogrammetric Engineering, v. 37, n.8, p. 855-866.
Campos, M., Tommaselli, A., Ivánová, I., Billen, R., 2015. Data product specification proposal for architectural heritage documentation with photogrammetric techniques: a case study in Brazil. Remote Sensing, 7(10), 13337-13363.

Campos, M. B., Tommaselli, A. M. G., Marcato Junior, J., Honkavaara, E., 2018. Geometric model and assessment of a dual-fisheye imaging system. The Photogrammetric Record, 33(162), 243-263. doi.org/10.1111/phor.12240.

Campos, M. B., Tommaselli, A. M. G., Castanheiro, L. F., Oliveira, R. A., Honkavaara, E., 2019. A Fisheye Image Matching Method Boosted by Recursive Search Space for Close Range Photogrammetry. Remote Sensing, v. 11, n. 12, p. 1404. doi.org/10.3390/rs11121404.

Caruso, D., Engel, J., Cremers, D., 2015. Large-scale direct slam for omnidirectional cameras. In: 2015 IEEE/RSJ International Conference on Intelligent Robots and Systems (IROS). doi.org/10.1109/IROS.2015.7353366

Conrady, A., 1919. Decentered lens systems. Monthly Notices of the Royal Astronomical Society, 79(5): 384-390.

Ericson, S., Åstrand, B., 2010. Row-detection on an agricultural field using omnidirectional camera. In 2010 IEEE/RSJ International Conference on Intelligent Robots and Systems, 4982-4987. doi.org/10.1109/IROS.2010.5650964.

Esau, T., Zaman, Q., Groulx, D., Farooque, A., Schumann, A., Chang, Y., 2018. Machine vision smart sprayer for spotapplication of agrochemical in wild blueberry fields. Precision agriculture, 19(4), 770-788. doi.org/10.1007/s11119-017-9557-

Fraser, C. S., 1997. Digital camera self-calibration. ISPRS Journal of Photogrammetry and Remote sensing, v. 52, n. 4, p. 149-159. doi.org/10.1016/S0924-2716(97)00005-1.

Kannala, J., Brandt, S. S., 2006. A generic camera model and calibration method for conventional, wide-angle, and fish-eye lenses. IEEE transactions on pattern analysis and machine intelligence, 28(8), 1335-1340.

Lehtola, V. V.; Kaartinen, H.; Nüchter, A.; Kaijaluoto, R.; Kukko, A.; Litkey, P.; Kurkela, M. Comparison of the selected state-of-the-art 3D indoor scanning and point cloud generation methods. Remote Sensing, v. 9, n. 8, p. 796, 2017.

Marcato Junior, J., Moraes, M. V. A., Tommaselli, A. M. G., 2015. Experimental assessment of techniques for fisheye camera calibration. Boletim de Ciências Geodésicas, v.21, n.3, p. 637651. doi.org/10.1590/S1982-21702015000300036

Moriya, É. A. S., Imai, N. N., Tommaselli, A. M. G., Berveglieri, A., Honkavaara, E., Soares, M. A., Marino, M., 2019. Detecting citrus huanglongbing in brazilian orchards using hyperspectral aerial images. In International Archives of the Photogrammetry, Remote Sensing and Spatial Information Sciences, XLII-2/W13, 1881-1886. doi.org/10.5194/isprs-archives-XLII-2-W13-18812019.

Ohi, N., Lassak, K., Watson, R., Strader, J., Du, Y., Yang, C., Hedrick, G., Nguyen, J., Harper, S., Reynolds, D., Kilic, C., Hikes, J., Mills, S., Castle, C., Buzzo, B., Waterland, N., Gross, J., Parks, Y. L., Li, X., Gu, Y., 2018. Design of an autonomous precision pollination robot. In 2018 IEEE/RSJ International 
Conference on Intelligent Robots and Systems (IROS), 77117718. doi.org/10.1109/IROS.2018.8594444.

Prosdocimi, M., Burguet, M., Di Prima, S., Sofia, G., Terol, E., Comino, J. R., Cerdà, A. Tarolli, P., 2017. Rainfall simulation and Structure-from-Motion photogrammetry for the analysis of soil water erosion in Mediterranean vineyards. Science of the Total Environment, 204-215. doi.org/10.1016/j.scitotenv.2016.09.036.

Ravi, R., Lin, Y. J., Shamseldin, T., Elbahnasawy, M., Crawford, M., Habib, A., 2018. Implementation of UAV-Based Lidar for High Throughput Phenotyping. In IGARSS 2018-2018 IEEE International Geoscience and Remote Sensing Symposium, 87618764. doi.org/10.1109/IGARSS.2018.8518439

Scaramuzza, D., Martinelli, A., Siegwart, R., 2006. A toolbox for easily calibrating omnidirectional cameras. In 2006 IEEE/RSJ International Conference on Intelligent Robots and Systems, 5695-5701. Doi.org/10.1109/IROS.2006.282372

Schneider, D., Schwalbe, E., Maas, H. G., 2009 Validation of geometric models for fisheye lenses. ISPRS Journal of Photogrammetry and Remote Sensing. v. 64, n.3, p. 259-266. doi.org/10.1016/j.isprsjprs.2009.01.001.

Sodhi, P., Vijayarangan, S., Wettergreen, D., 2017. In-field segmentation and identification of plant structures using $3 \mathrm{D}$ imaging. In 2017 IEEE/RSJ International Conference on Intelligent Robots and Systems (IROS) (pp. 5180-5187).

Song, W., Liu, X., Lu, P., Huang, Y., Weng, D., Zheng, Y., Liu, Y., Wang, Y., 2018. Design and assessment of a $360^{\circ}$ panoramic and high-performance capture system with two tiled catadioptric imaging channels. Applied optics, v. 57, n. 13, p. 3429-3437. doi.org/10.1364/AO.57.003429

Tommaselli, A. M. G.; Marcato Junior, J.; Moraes, M. V. A.; Silva, S. L. A.; Artero, A. O., 2014. Calibration of panoramic cameras with coded targets and a 3D calibration field. The International Archives of Photogrammetry, Remote Sensing and Spatial Information Sciences, XL-3, p. 137. doi.org/10.5194/isprsarchives-XL-3-W1-137-2014

Zhang, Z., Rebecq, H., Forster, C., Scaramuzza, D., 2016. Benefit of large field-of-view cameras for visual odometry. In 2016 IEEE International Conference on Robotics and Automation (ICRA), pp. 801-808. doi.org/10.1109/ICRA.2016.7487210 